\title{
Anatomical variations of the hepatic artery: a closer view of rare unclassified variants
}

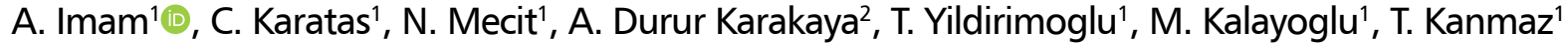 \\ ${ }^{1}$ Liver Transplantation Department, Koc University Hospital, Istanbul, Turkey \\ ${ }^{2}$ Radiology Department, Koc University Hospital, Istanbul, Turkey
}

[Received: 21 August 2020; Accepted: 1 February 2021; Early publication date: 1 March 2021]

\begin{abstract}
Background: Defining the hepatic artery anatomy is of great importance for both surgeons and radiologists. Michel classification was designed to classify hepatic artery variations. Nevertheless, there are variations that do not fit into this classification. In this study, we aim to define the incidence of all variations in a healthy liver donor by reviewing their computed tomography (CT) scan with special emphasis on variations that do not fit in any of the Michel classes.

Materials and methods: A retrospective analysis of CT scan of donors and potential liver donors who were evaluated by triphasic CT scan. The CT scans were reviewed independently by a radiologist and two transplant surgeons. Cases that did not fit in any of the Michel classes were classified as class 0.

Results: Out of 241 donors, 210 were classified within the Michel classification, of which $60.9 \%$ were class I and $9.1 \%$ class II. Thirty-one (12.9\%) donors classified as class 0 . Of which, nine, three, two and three had replaced right hepatic artery from pancreaticoduodenal artery, gastroduodenal artery, aorta and coeliac artery, respectively. Two and six donors had accessory right hepatic artery from pancreaticoduodenal artery and gastroduodenal artery, respectively. Segment 4 artery originated from left and right hepatic artery in $56.8 \%$ and $31.9 \%$, respectively. Conclusions: A great caution should be taken when evaluating the hepatic artery anatomy, clinicians should anticipate and be familiar with the rare unclassified variations of the hepatic artery. (Folia Morphol 2022; 81, 2: 359-364)
\end{abstract}

Key words: anatomical variations, computed tomography, hepatic artery, replaced right hepatic artery, gastroduodenal artery, pancreaticoduodenal artery

\section{INTRODUCTION}

The anatomical variation of the coeliac trunk and its branches may affect the surgical approach of several organs such as stomach, duodenum, pancreas, gallbladder and most importantly the liver [16]. Hepatic artery variations were described in 55\% of cadavers by Michel [see 5].
In 1995, Michel classification was established, with 10 different classes that described the hepatic artery anatomy and its variations [11]. These variations are of great importance for the transplant, laparoscopic, hepatobiliary, trauma surgeons and interventional radiologists [1, 13]. There are several reports in the literature regarding hepatic artery variations that do not fit in Michel classification $[3,6,8,10]$.

Address for correspondence: A. Imam, MD, Topkapı, Koç Üniversitesi Hastanesi, Davutpaşa Cd. No:4, Zeytinburnu/İstanbul, 34010, Turkey, tel: +90 8502508 250, fax: +90212 31134 10, e-mail: ash_imam04@hotmail.com

This article is available in open access under Creative Common Attribution-Non-Commercial-No Derivatives 4.0 International (CC BY-NC-ND 4.0) license, allowing to download articles and share them with others as long as they credit the authors and the publisher, but without permission to change them in any way or use them commercially. 
Table 1. The distribution of cases in each class of Michel classification and the segment 4 artery origin in each class

\begin{tabular}{|c|c|c|c|c|c|c|c|c|c|c|c|}
\hline & \multicolumn{11}{|c|}{ Class } \\
\hline & I & II & III & IV & V & VI & VII & VIII & IX & $\mathrm{X}$ & $0^{*}$ \\
\hline Number & 147 & 22 & 18 & 1 & 11 & 1 & 1 & 7 & 2 & 0 & 31 \\
\hline S4 artery - RHA & 50 & 13 & 1 & 0 & 6 & 1 & 1 & 1 & 0 & 0 & 4 \\
\hline S4 artery — LHA & 84 & 7 & 17 & 1 & 5 & 0 & 0 & 5 & 2 & 0 & 16 \\
\hline S4 artery - PHA & 0 & 1 & 0 & 0 & 0 & 0 & 0 & 0 & 0 & 0 & 1 \\
\hline S4 artery - Coeliac & 0 & 0 & 0 & 0 & 0 & 0 & 0 & 0 & 0 & 0 & 4 \\
\hline S4 artery not seen & 11 & 1 & 0 & 0 & 0 & 0 & 0 & 1 & 0 & 0 & 6 \\
\hline Other $\$ 4$ artery & $\mathrm{L}$ and $\mathrm{R} S 4$ artery & 0 & 0 & 0 & 0 & 0 & 0 & 0 & 0 & 0 & AS4-GDA \\
\hline
\end{tabular}

*The cases that did not fit in any of the Michel classes. S4 artery — segment 4 hepatic artery; RHA — right hepatic artery; LHA — left hepatic artery; PHA — proper hepatic artery; $\mathrm{L}$ — left; $\mathrm{R}$ — right; AS4 — accessory segment 4 artery; GDA — gastroduodenal artery

In this article, we describe the anatomical variation of the hepatic artery of donors and potential liver donors that had a triphasic computed tomography (CT) scan for anatomical evaluation, with special emphasis on variations that were not described by Michel.

\section{MATERIALS AND METHODS}

Institutional review board approval was obtained and waived the need for informed consent. A retrospective analysis of prospectively collected data of all liver donors and potential donors during a 2 years period in our transplant centre. All the arterial vascular anatomy was prospectively collected.

The CT scans of the donors were independently reviewed by two transplant surgeons and a senior radiologist using Myrian studio software by Intrasene.

\section{The CT protocol}

The donors were subjected to a triple phase multidetector CT scan using Siemens SOMATOM Definition Flash. An un-enhanced, hepatic venous, arterial and portal venous phases were obtained.

At a rate of $3.0 \mathrm{~mL} / \mathrm{s}$, a $1.5 \mathrm{~mL} / \mathrm{kg}$ of $300 \mathrm{mg} / \mathrm{mL}$ non-ionic iodinated contrast using a pressure injector was administered intravenously with an 18-20 gauge peripheral access catheter in the arm to all donors. After setting the trigger to a 100 Hounsfield Unit, the region of interest cursor was placed in the aorta just above the dome of the diaphragm.

The unenhanced and hepatic venous phases included the whole abdominal organ while the arterial, and portal phases were directed to the liver.

\section{Arterial classification}

Donors who had a low-quality CT scan were excluded from the study.
The patients were classified according to Michel classification. Those who did not meet any of Michel classification subtypes were classified as class 0 . Additional detailed description of the class 0 type was given.

\section{RESULTS}

Out of the 263 donors and potential liver donors that have been evaluated in the study period, 241 were included in the study. The remaining 22 donors were excluded due to low quality CT scan. 210 patients met Michel classification subtypes of which 147 (60.9\%) were class I which is the most common. The second most common variation was class II with 22 (9.1\%) donors, followed by class III, 18 (7.5\%) donors. Table 1 demonstrates the number of patients in all classes.

Thirty-one (12.9\%) donors did not fit in any of the classes of Michel classification and were classified as class 0 (Table 2). Of which, 9, 2, 3 and 6 had replaced right hepatic artery (RRHA) from pancreaticoduodenal artery (PDA) (Fig. 1), accessory right hepatic artery (ARHA) from PDA, RRHA from gastroduodenal artery (GDA) (Fig. 2), ARHA from GDA, respectively (Table 3). Two donors had an RRHA directly from the aorta (Figs. 3, 4), three donors had RRHA from the coeliac artery (Fig. 5), and one had ARHA from coeliac artery. Additional variations include two left hepatic arteries (LHA) from the proper hepatic artery (PHA) which was demonstrated in five donors. One case had a hepatic trunk that originated from the superior mesenteric artery (SMA) with accessory LHA from the left gastric artery and another case had an accessory segment four artery from the GDA. Table 2 demonstrates class 0 variation in detail.

It's worth mentioning that some donors share more than one unclassified variation. 
Table 2. The characteristic of all class 0 (unclassified) cases

\begin{tabular}{|c|c|c|c|c|c|c|c|c|c|}
\hline & RHA-PHA & ARHA-PDA & RRHA-PDA & ARHA-GDA & RRHA-GDA & RRHA-Coeliac & ARHA-Coeliac & RRHA-Aorta & Others \\
\hline LHA-PHA & 0 & 1 & $4^{*}$ & $3^{*}$ & 1 & 2 & 1 & 0 & 0 \\
\hline ALHA-LGA & 0 & 0 & 1 & 1 & 2 & 0 & 0 & 0 & 0 \\
\hline RLHA-LGA & 0 & 1 & 3 & 2 & 0 & 1 & 0 & 2 & 0 \\
\hline Two LHA-PHA & 4 & 0 & 1 & 0 & 0 & 0 & 0 & 0 & 0 \\
\hline Other & 0 & 0 & 0 & 0 & 0 & 0 & 0 & 0 & 2 \\
\hline
\end{tabular}

${ }^{*}$ One patient had ARHA from GDA (small branch) and RRHA from PDA (main branch). LHA — left hepatic artery; RHA — right hepatic artery; PHA — proper hepatic artery;

LGA — left gastric artery; ALHA — accessory left hepatic artery; RLHA — replaced left hepatic artery; ARHA — accessory right hepatic artery; RRHA — replaced right hepatic artery; PDA — pancreaticoduodenal artery; GDA — gastroduodenal artery

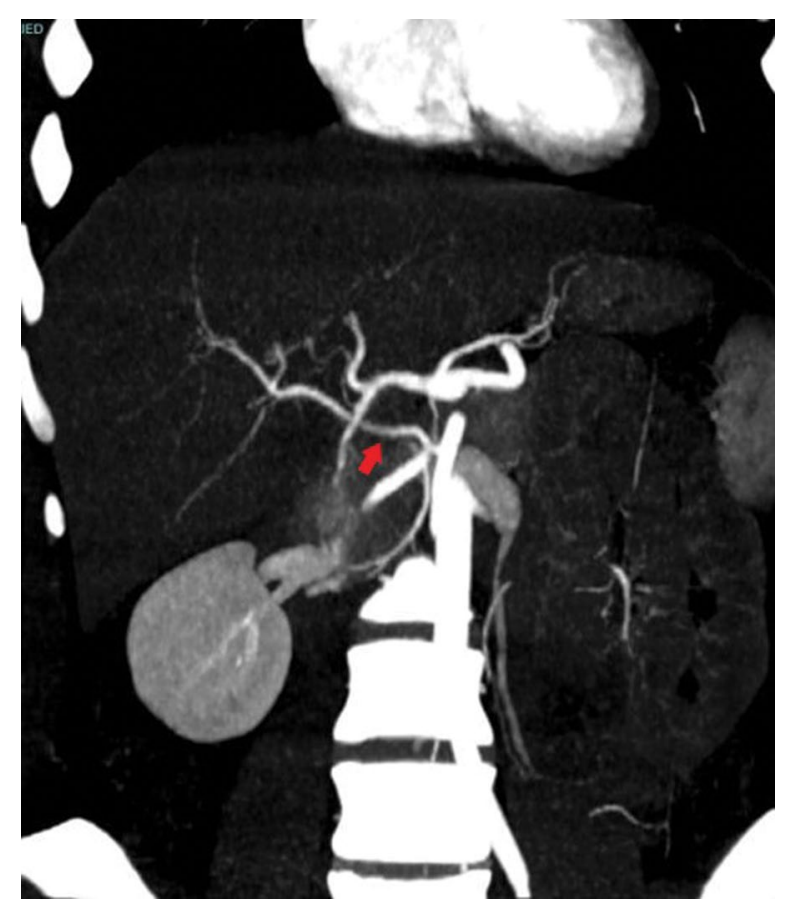

Figure 1. Computed tomography three-dimensional view: replaced right hepatic artery from pancreaticoduodenal artery (red arrow).

Segment 4 artery (S4) was also studied (Tables 1,3$)$. In 19 patients, S4 could not be visualised.

In general, $137(56.8 \%)$ donors had S4 from the LHA, 77 (31.9\%) from the right hepatic artery (RHA), 2 from the PHA, and four from the coeliac artery. One donor had a S4 from both LHA and RHA. And one case had accessory $\$ 4$ from the GDA.

\section{DISCUSSION}

The standard hepatic artery anatomy with the left and right hepatic artery arising from the common hepatic artery after giving of the gastroduodenal artery is present in up to $55 \%$ of the population [11].

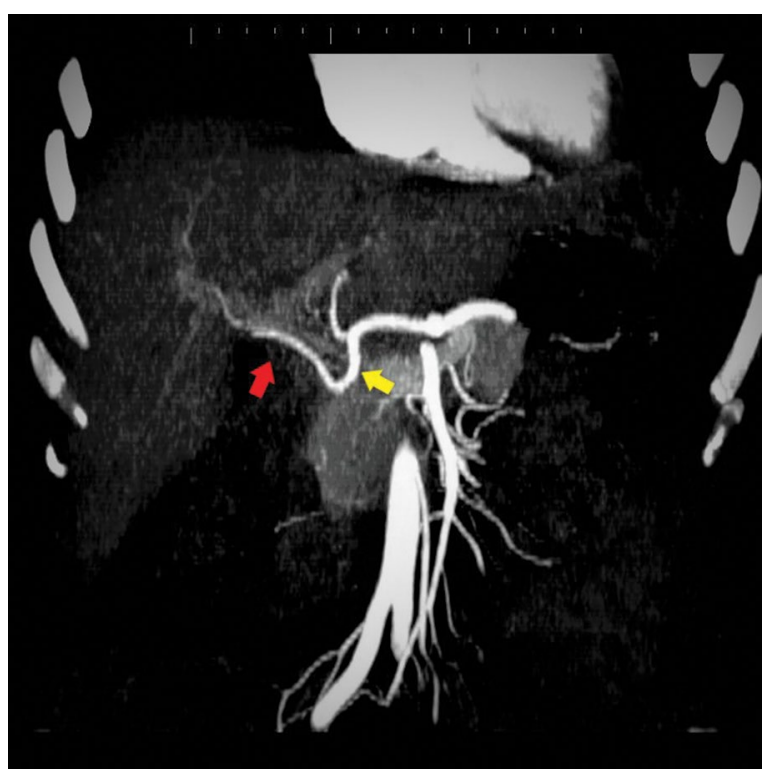

Figure 2. Computed tomography coronal view: replaced right hepatic artery (red arrow) from the gastroduodenal artery (yellow arrow).

Determining the hepatic artery variation is of utmost importance for the surgeon and the interventional radiologist [1, 13].

In our study, we found that the standard anatomy is present in $60.9 \%$ of the healthy donors, which is similar to what was previously described in the literature [4].

From embryological point of view, there are four primitive splanchnic branches arises from aorta early during the embryo development. Those branches are connected by longitudinal anastomosis in their roots. Some of the branches disappear later during the embryo development. The common hepatic artery originates from this longitudinal anastomosis. Disappearance or retention of parts of this plexus could give numerous anatomical variations $[12,17]$. 
Table 3. The anatomical details of replaced and accessory right hepatic artery cases that originate from the gastroduodenal artery

\begin{tabular}{|c|c|c|c|c|c|c|c|c|}
\hline & ARHA-GDA & RRHA-GDA & LHA-PHA & ALHA-LGA & RLHA-LGA & S4-L & S4-R & S4 not seen \\
\hline 1 & * & & & & * & & * & \\
\hline 2 & $*$ & & & & $*$ & & $*$ & \\
\hline 3 & * & & & $*$ & & $*$ & & \\
\hline 4 & * & & $*$ & & & & & $*$ \\
\hline 5 & $*$ & & $*$ & & & & & * \\
\hline 6 & & $*$ & & * & & & & * \\
\hline 7 & & $*$ & & * & & $*$ & & \\
\hline 8 & & * & & & & $*$ & & \\
\hline
\end{tabular}

LHA — left hepatic artery; PHA — proper hepatic artery; LGA — left gastric artery; ALHA — accessory left hepatic artery; RLHA — replaced left hepatic artery; ARHA — accessory right hepatic artery; RRHA — replaced right hepatic artery; GDA — gastroduodenal artery; S4-L — segment 4 artery from the left hepatic artery; S4-R — segment 4 artery from the right hepatic artery

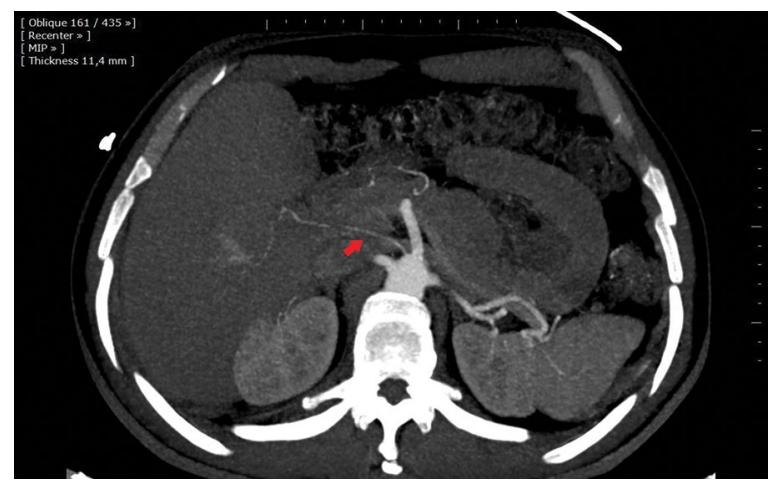

Figure 3. Computed tomography axial view: replaced right hepatic artery from the aorta (red arrow).

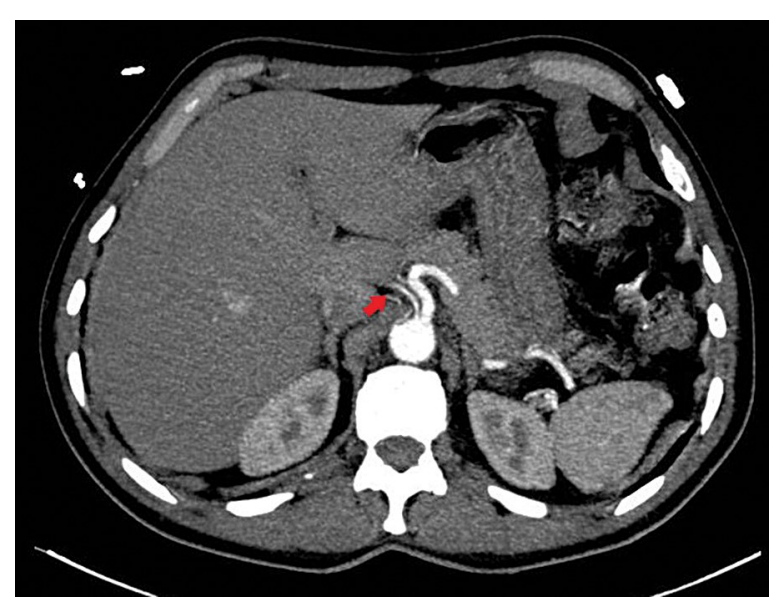

Figure 4. Computed tomography axial view: replaced right hepatic artery from the aorta (red arrow) (additional view).

One of the most widely accepted classification of the hepatic artery anatomical variation is Michel classification, which includes 10 classes [11]. In our

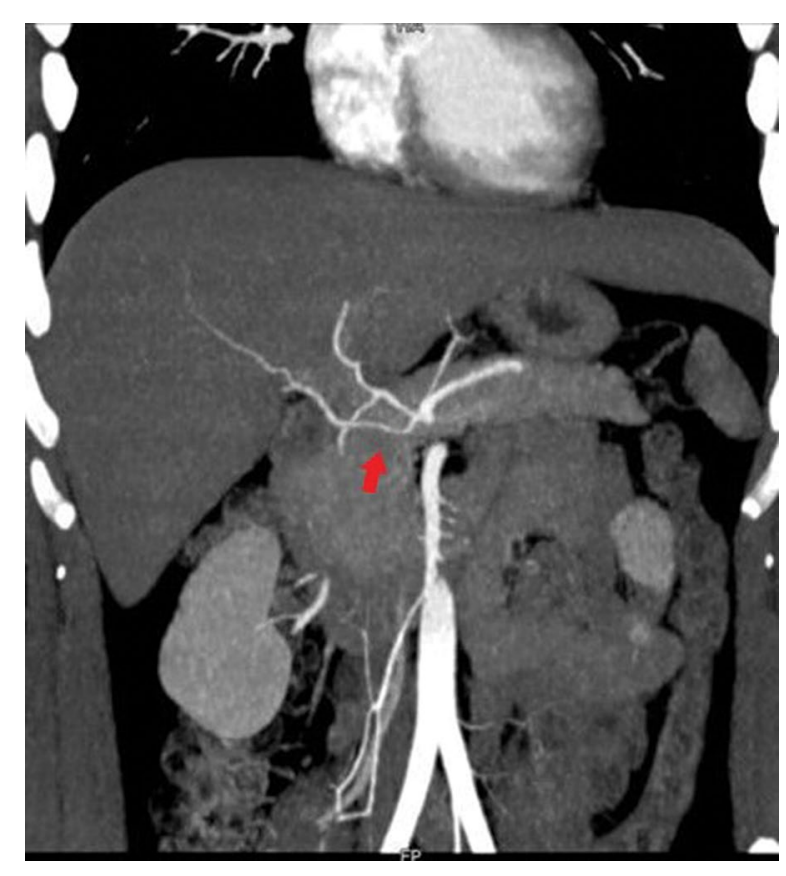

Figure 5. Computed tomography coronal view: replaced right hepatic artery from the coeliac artery (red arrow).

study, $12.9 \%$ of the population did not fit in any of the classes of Michel classification and were classified as class 0 . This relatively high incidence shows the value of identifying those variants. For example, in our sample we found that $9(3.7 \%)$ cases have either replaced or accessory RHA from the GDA, these variations were barely mentioned in the literature and were limited to case reports [15]. Identifying these findings may be critical in the setting of liver transplantation when performing liver procurement, since this branch can be preserved by harvesting the GDA with its branches and keeping the RRHA or 
ARHA intact. Thus, minimizing the number of arterial anastomosis and, in turn, decreasing the chance of arterial complications.

Furthermore, this variation may be of great importance for interventional radiologists especially when performing trans-arterial chemoembolisation for hepatocellular carcinoma, since this aberrant branch may feed the tumour [9]. Moreover, a great caution should be taken when embolising the GDA as a result of bleeding, due to the risk of cutting off the blood supply to the right segments of the liver in case of RRHA.

Another variation that is usually misclassified as class III or VI is when the RRHA or ARHA originate from the PDA or when they both share a common trunk. The importance of this variation arises when performing pancreaticoduodenectomy or when performing angiographic embolisation of the rare PDA pseudoaneurysm [7]. In case of pancreaticoduodenectomy as a result of a tumour, it is important to identify the arterial variations and their relation to the tumour in order to perform a safe surgery and to avoid vascular complications [14]. Alternatively, it may be better to subclassify this variant as class III $p$ or class VI $p$ when the RRHA or ARHA originate from the PDA.

An additional variant is the RRHA directly from the aorta, this variant may be important when performing angiography and trans-arterial chemoembolisation for hepatocellular carcinoma [2], since it may be challenging to access this artery. Similarly, the significance of this variation emerges during liver harvest in the setting of cadaveric donation because it may be confusing to the surgeon if he is not familiar with this rare variation.

Another rare variation is a case that had a hepatic trunk that originated from the SMA with accessory LHA from the left gastric artery, this variation may make pancreaticoduodenectomy procedure more challenging and may increase the vascular complication rate as was reported by Ye et al. [18].

In this study, we also focused on identifying the root of the S4 hepatic artery (middle hepatic artery). Turning back to the literature including Michel classification, limited data is documented regarding the segment 4 arterial supply, and Michel classification didn't include the $\$ 4$ artery variation. It is worth noting that $\mathrm{S} 4$ hepatic artery determination is very important in the setting of live donor liver donation. For instance, when performing left lateral segmentectomy, there might be a high probability of sacrificing it in case it arises from the left hepatic artery and, in turn, a higher chance of complications to the donor. On the contrary, when a right hepatectomy is to be performed, the arterial blood supply to segment 4 may be affected if the $\mathrm{S} 4$ artery originates from the right hepatic artery [4].

We believe that adding an additional class to Michel classification, with class 0 as a non-conventional variation, may help both the radiological and surgical team to develop a common language and make the surgeon or the interventional radiologist more alert, and fully prepared for these anatomical variations during the procedure.

A limitation of this study is that it included both donors and potential donors so a correlation with operative findings is not possible. An additional limitation is that it did not include the hepatic duct or portal vein variations.

\section{CONCLUSIONS}

A meticulous pre-procedure imaging revision for identification of the hepatic artery variation and keeping in mind the possibility of non-conventional variations that were described in this study is a key for performing a successful procedure with minimal complications.

\section{Acknowledgements}

The assistance provided by Riham Imam in technical editing of the manuscript is greatly appreciated.

\section{Conflict of interest: None declared}

\section{REFERENCES}

1. Balachandran A, Darden DL, Tamm EP, et al. Arterial variants in pancreatic adenocarcinoma. Abdom Imaging. 2008; 33(2): 214-221, doi: 10.1007/s00261-007-9235-z, indexed in Pubmed: 17435979.

2. Cazejust J, Bessoud B, Colignon N, et al. Hepatocellular carcinoma vascularization: from the most common to the lesser known arteries. Diagn Interv Imaging. 2014; 95(1): 27-36, doi: 10.1016/j.diii.2013.04.015, indexed in Pubmed: 23978434.

3. Ciyiltepe H, Cetin D, Aday U, et al. Rare hepatic artery anomaly (Michel type unclassified) in a patient who underwent radical gastrectomy for gastric cancer: a case report. Bezmialem Science. 2017; 5(3): 142-144, doi: 10.14235/bs.2017.1104.

4. Favelier S, Germain T, Genson PY, et al. Anatomy of liver arteries for interventional radiology. Diagn Interv Imaging. 2015; 96(6): 537-546, doi: 10.1016/j.diii.2013.12.001, indexed in Pubmed: 24534562.

5. Goss C. Blood supply and anatomy of the upper abdominal organs with a descriptive atlas. By N.A. Michels. xiv +581 
pages, 172 figures. $\$ 24.00$. J. B. Lippincott Company, Philadelphia, 1955. Anat Rec. 1960; 137(2): 153-154, doi: 10.1002/ar.1091370211.

6. Hlaing KP, Othman F. Complex pattern of a variant hepatic artery. Singapore Med J. 2012; 53(9): e186-e188, indexed in Pubmed: 23023911.

7. Imam R, Khalayleh H, Khoury D, et al. Rupture of an idiopathic aneurysm of the inferior pancreaticoduodenal artery leading to an intra-abdominal bleeding. J Acute Care Surg. 2020; 10(1): 33-35, doi: 10.17479/jacs.2020.10.1.33.

8. Johnson PB, Cawich SO, Shah S, et al. Vascular supply to the liver: a report of a rare arterial variant. Case Rep Radiol. 2013; 2013: 969327, doi: 10.1155/2013/969327, indexed in Pubmed: 24159405.

9. Liang $Y, L i E$, Min J, et al. Rare anatomic variation of the right hepatic artery and accessory right hepatic artery supplying hepatocellular carcinoma: A case report and literature review. Medicine (Baltimore). 2017; 96(39): e8144, doi: 10.1097/ MD.0000000000008144, indexed in Pubmed: 28953651.

10. Mehta V, Dave V, Suri RK, et al. Quadrifurcation of the hepatic artery proper in conjunction with double right gastric arteries. Singapore Med J. 2012; 53(10): e211e213, indexed in Pubmed: 23112032.

11. Michels NA. Newer anatomy of the liver and its variant blood supply and collateral circulation. Am J Surg. 1966; 112(3): 337-347, doi: 10.1016/0002-9610(66)90201-7, indexed in Pubmed: 5917302.

12. Morita M. Reports and conception of three anomalous cases on the area of the celiac and the superior mesenteric arteries. Igaku Kenkyu. 1936; 5: 159-172.
13. Munshi IA, Fusco D, Tashjian D, et al. Occlusion of an aberrant right hepatic artery, originating from the superior mesenteric artery, secondary to blunt trauma. Trauma. 2000; 48(2): 325-326, doi: 10.1097/00005373200002000-00025, indexed in Pubmed: 10697098.

14. Padilla Valverde $D$, Villarejo Campos $P$, Villanueva Liñán J, et al. [Radiological-surgical methods to identify celiac-mesenteric anomalies of the hepatic artery before duodenopancreatectomy]. Cir Esp. 2013; 91(2): 103-110, doi: 10.1016/j.ciresp.2012.04.012, indexed in Pubmed: 23219204.

15. Polguj $M$, Podgórski $M$, Hogendorf $P$, et al. Variations of the hepatobiliary vasculature including coexistence of accessory right hepatic artery with unusually arising double cystic arteries: case report and literature review. Anat Sci Int. 2014; 89(3): 195-198, doi: 10.1007/s12565013-0219-5, indexed in Pubmed: 24310410.

16. Prakash, Rajini T, Mokhasi V, et al. Coeliac trunk and its branches: anatomical variations and clinical implications. Singapore Med J. 2012; 53(5): 329-331, indexed in Pubmed: 22584973.

17. Tandler J. Über die Varietäten der Arteria coeliaca und deren Entwickelung. Beiträge und Referate zur Anatomie und Entwickelungsgeschichte. 1904; 25(2): 473-500, doi: 10.1007/bf02300762.

18. Ye Z, Ye S, Zhou D, et al. A rare variation of celiac trunk and hepatic artery complicating pancreatico-duodenectomy: A case report and literature review. Medicine (Baltimore). 2017; 96(48): e8969, doi: 10.1097/ MD.0000000000008969, indexed in Pubmed: 29310402. 